\title{
Genetic diversity analysis of traditional and improved cultivars of Pakistani rice (Oryza sativa L.) using RAPD markers
}

\author{
Malik Ashiq Rabbani* \\ Institute of Agri-Biotechnology \& Genetic Resources \\ National Agricultural Research Center \\ Park Road, Islamabad, Pakistan \\ Tel: 92519255199 \\ Fax: 92519255201 \\ E-mail: rabbani316@yahoo.com \\ Zahida Hassan Pervaiz \\ Department of Biochemistry \\ Quaid-i-Azam University \\ Islamabad, Pakistan \\ Tel: 923075383717 \\ E-mail: zahidahasan82pk@yahoo.co.uk \\ Muhammad Shahid Masood \\ Institute of Agri-Biotechnology \& Genetic Resources \\ National Agricultural Research Center \\ Park Road, Islamabad, Pakistan \\ Tel: 92519255199 \\ Fax: 92519255201 \\ Email: shahid617@yahoo.com
}

\begin{abstract}
Financial support:This work was carried out under Agricultural Linkages Program. Authors are grateful to Pakistan Agricultural Research Council for the financial support from Agricultural Linkages Program under the AREF.
\end{abstract}

Keywords: cultivar discrimination, genetic variability, Oryza sativa, Pakistani rice, RAPDs.

Abbreviations: AREF: Agricultural Research Endowment Fund

RAPD: Random Amplified Polymorphic DNA

UPGMA: unweighted pair-group method with arithmetic averages

The molecular marker is a useful tool for assessing genetic variations and resolving cultivar identities. Information on genetic diversity and relationships among rice genotypes from Pakistan is currently very limited. The objective of this study was to evaluate the genetic polymorphisms and identities of 10 traditional, 28 improved and 2 Japanese cultivars of rice using the random amplified polymorphic DNA technique. Twenty-five decamer-primers could generate a total of 208 RAPD fragments, of which 186 or $89.4 \%$ were polymorphic. The number of amplification products produced by each primer varied from 4 to 16 with an average of 8.3 bands primer $^{-1}$. The size of amplified fragments were ranged from 200 to 4000 bp. Pair-wise Nei and Li's similarity had estimated the range of 0.50 to 0.96 between rice cultivars. Based on analysis performed on a similarity matrix using UPGMA, 40 cultivars were grouped into 3 main clusters corresponding to aromatic, non-aromatic and japonica group. There were a few of independent cultivars. The cluster analysis had placed most of the aromatic cultivars into a close relation showing a high level of genetic relatedness. However, the clusters formed by the aromatic cultivars were distinct from those of nonaromatic and japonica types. Interestingly, a number of improved and traditional cultivars originating from diverse sources did not form well defined groups and were interspersed, indicating no association between the RAPD patterns and the geographic origin of the cultivars. The information generated from this study can be used to maximize selection of diverse parents and broaden the germplasm base in the future of rice breeding programs.

Rice (Oryza sativa L.) is one of the most important crops that provide food for more than half of the world population. It is no longer a luxury food but has become the cereal that constitutes a major source of calories for the urban and the rural (Sasaki and Burr, 2000). Rice is cultivated in a wide range of ecological environments worldwide. Pakistan is one of the few countries which had an enormous wealth of aromatic rices and old landraces. Many have been lost during the last three decades after green revolution. A number of traditional varieties and improved cultivars have been released for cultivation in different regions of Pakistan since the early nineteen

*Corresponding author 

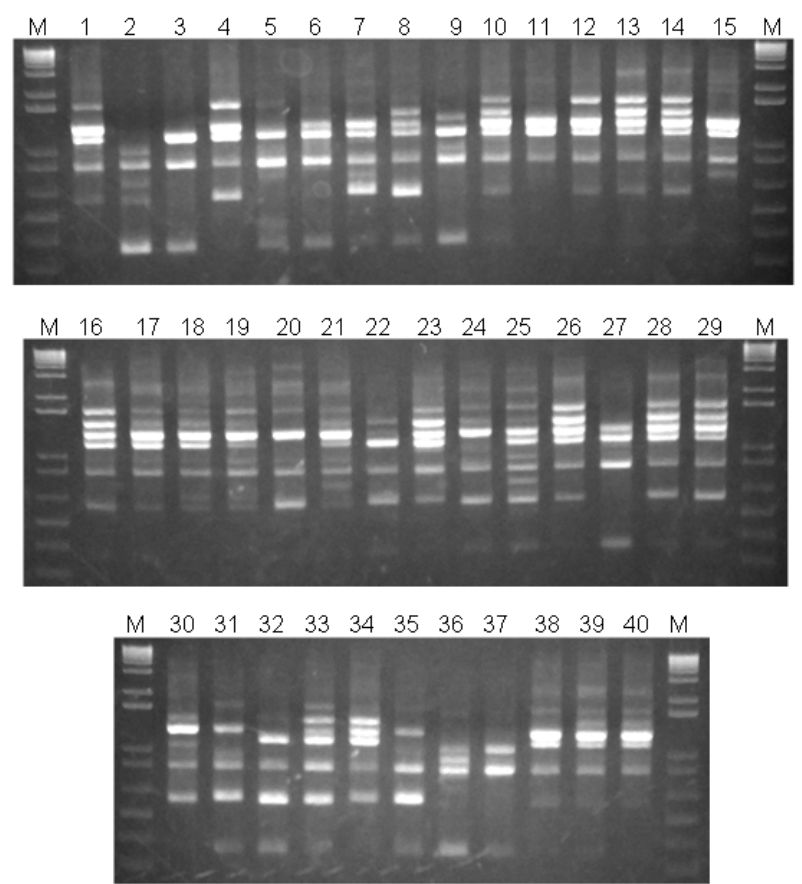

Figure 1. RAPD banding pattern of 40 traditional and improved cultivars of Pakistani rice generated by random primer OPA-18. The lanes represent M-1Kb plus molecular size marker; 1-Basmati-370, 2-Jhona-349, 3-Shaheen-basmati, 4Palman-sufaid, 5-Basmati-C622, 6-Basmati-Pak, 7-Pak23710, 8KS-282, 9-Basmati-385, 10-Super-basmati, 11-Basmati-2000, 12-Kashmir-basmati, 13-NIAB-IR9, 14-Sathra, 15-Rachnabasmati, 16-IR6, 17-Sugdasi-ratria, 18-Jajai-77, 19-Kangni-27, 20-DR-82, 21-DR-83, 22-Lateefy, 23-Sada-hayat, 24-DR-92, 25Kanwal-95. 26-Shua-92, 27-Khushboo-95, 28-Shadab, 29Sarshar, 30-Swat-1, 31-Swat-2, 32-Pakhal. 33-Dilrosh-97, 34Fakhre-Malakand, 35-IR36, 36-Kinmaze, 37-Nipponbare, 38Sugdasi-bengalo, 39-Sugdasi-sadagulab, 40-Sonahri-sugdasi.

century. Aromatic varieties and advanced breeding lines have narrowed the genetic basis as they are being evolved from the selected basmati lines. Also very limited number of basmati breeding lines are being used repeatedly in the breeding programme. It only contributed to the grain quality but not to the resistance against biotic and abiotic stresses. In Pakistan, out of 7 basmati varieties were currently under cultivation, 5 had 'Basmati-370' as one of the parent cultivar (Arif et al. 2005). There is a strong need to not only conserve landrace genotypes but also broaden the gene-pool of aromatic rice for the future utilization in breeding of high yielding, superior quality and betteradapted varieties in the country.

Identification of different genotypes of crop species is essential when diverse accessions of crop germplasm are to be characterized, newly developed cultivar is to be registered and purity of the variety is to be determined. Among numerous techniques available for assessing the genetic variability and relatedness among crop germplasm, DNA-based markers provide very effective and reliable tools for measuring genetic diversity in crop germplasm and studying evolutionary relationships. Molecular genetic techniques using DNA polymorphism have been increasingly used to characterize and identify a novel germplasm for uses in the crop breeding process (O'Neill et al. 2003). Unlike the morphological and biochemical markers which may be affected by environmental factors and growth practices, DNA markers depict genome sequence composition, thus enabling the detection of differences in the genetic information carried by the different individuals.

Among the molecular markers, random amplified polymorphic DNA (RAPD) is increasingly being employed in genetic research owing to its speedy process and simplicity (Williams et al. 1990). RAPD requires no prior sequence information for the fingerprinting of cultivar genomes and it has been used extensively for estimating genetic variations at the population level and among closely related species. RAPD has been used in the analysis of rice genotypes by several groups for (i) estimation of the genetic diversity and relatedness among different accessions (Davierwala et al. 2000; Raghunathachari et al. 2000; Porreca et al. 2001; Neeraja et al. 2002; Saker et al. 2005); (ii) detection of duplicates (Virk et al. 1995; Verma et al. 1999); (iii) identification and classification of various cultivars (Ko et al. 1994; Mackill, 1995; Choudhury et al. 2001) and (iv) determination of the genetic relationship between several species (Bautista et al. 2001; Ren et al. 2003).

Although a large number of aromatic and course cultivars of rice are available in Pakistan, no systematic analysis has been carried out so far for genetic diversity, identification and discrimination except the previous report (Arif et al. 2005). In the present study, the evaluation of the pattern and the extence of genetic variability and relatedness among traditional varieties to improved cultivars of rice were conducted using RAPD markers. This would help in the identification and differentiation of various cultivars being cultivated and/or for exports. This will also contribute to maximize the selection of diverse parent cultivar and to broaden the germplasm base in the future of rice breeding programs. The information generated from this study will be used in identifying efficient strategies for the sustainable management of the genetic resources of rice crops.

\section{MATERIALS AND METHODS}

\section{Plant material}

A total of 40 traditional and improved cultivars of rice were used in this study. The research material represented the major rice varieties currently growing in various regions of the country as well as old cultivars whose cultivation was discontinued or banned because of their susceptibility to biotic and/or abiotic stresses. Among cultivars used, 16 belonged to aromatic (Basmati) group, 22 belonged to nonaromatic (coarse), while 2 japonica type cultivars from 


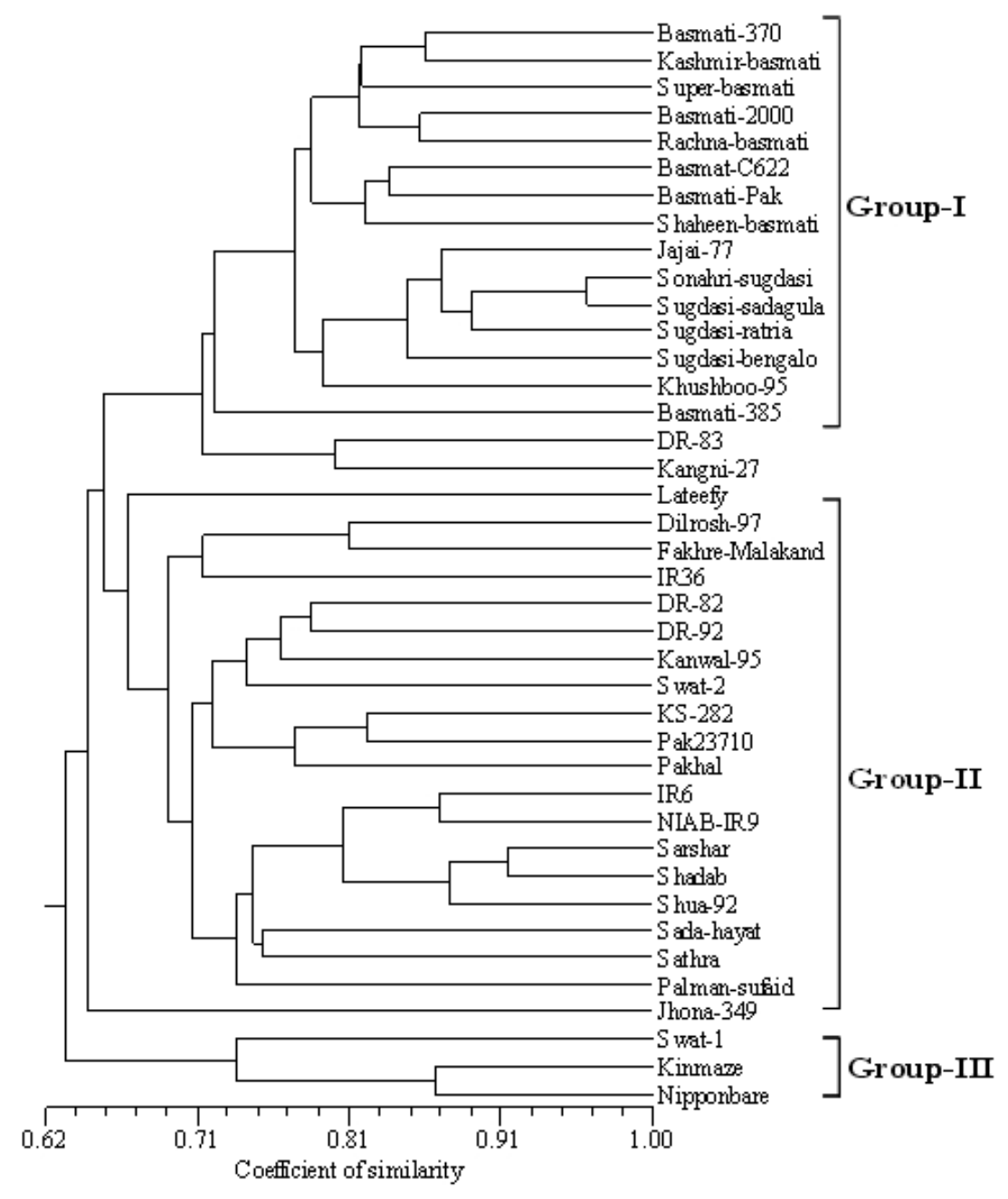

Figure 2. UPGMA cluster analysis showing the relationship and diversity among traditional and improved cultivars of rice based on 208 RAPD fragments produced by 25 random primers.

Japan were included for comparison. A detailed description of the materials used in present investigation is shown in Table 1.

\section{Genomic DNA Isolation}

Total genomic DNA was extracted from dry seed of ten individual plants of each cultivar according to the method described with minor modifications (Kang et al. 1998). Purity and concentration of DNA was monitored spectrophotometrically at a wavelength of 260 and $280 \mathrm{~nm}$ using NanoDrop ND-1000 Spectrophotometer. All the DNA samples were diluted to a working concentration of $20 \mathrm{ng} / \mu \mathrm{l}$. An equal amount of DNA from 10 individuals of each cultivar was mixed to make a bulk sample for PCR analysis.

\section{Primer selection and RAPD analysis}

Initially, 3 individual sample of each cultivar: aromatic, non-aromatic and japonica groups were used to select the suitable primers of which had exhibited variations. In total, 40 decamers of oligonucleotides belonging to kit OPA and OPB from Operon Technologies Inc. (Alameda, California, USA) were tested as single primers to identify the most promising detectable polymorphisms. After an initial screening, 32 primers were chosen on the basis of their ability to detect the polymorphisms and production of the reliable and scorable banding patterns in rice cultivars. Seven primers failed to amplify the DNA from some cultivars, therefore 25 primers could be used to show the clearly and consistently banding patterns. The best RAPD amplification pattern of each cultivar was ultimately chosen for data compilation and examination of the genetic diversity and relationship.

RAPD analysis was performed following the previous protocol with minor modification (Williams et al. 1990). Amplification reactions were carried out in a volume of 20 $\mu \mathrm{l}$ containing $10 \mathrm{mM}$ Tris $\mathrm{HCl}$ (pH 8.3), $50 \mathrm{mM} \mathrm{KCl,} 1.5$ $\mathrm{mM} \mathrm{MgCl}_{2}, 200 \mu \mathrm{M}$ each deoxynucleotide triphosphate (dNTP), $0.4 \mu \mathrm{M}$ of 10 - mer primer (Operon Technologies 
Table 1. Traditional and improved cultivars of Pakistani rice used in present study.

\begin{tabular}{|c|c|c|c|c|c|}
\hline No. & Cultivar Name & Parentage & Varietal group & Year & Breeding Institute ${ }^{*}$ \\
\hline 1 & Basmati-370 & Local selection & Aromatic & 1933 & RRI, KSK, Lahore \\
\hline 2 & Basmati-385 & Basmati-370/TN1 & Aromatic & 1988 & RRI, KSK, Lahore \\
\hline 3 & Basmati-2000 & Basmai-385/Super-Basmati & Aromatic & 2000 & RRI, KSK, Lahore \\
\hline 4 & Basmati-C622 & Basmati-370/Mushkan-7 & Aromatic & 1964 & RRI, KSK, Lahore \\
\hline 5 & Basmati-Pak & CM7-6/Basmati-370 & Aromatic & 1968 & RRI, KSK, Lahore \\
\hline 6 & Jajai-77 & Pure line selection & Aromatic & 1932 & RRI, Dokri, Sindh \\
\hline 7 & Kashmir-basmati & Mutant of Basmati-370 & Aromatic & 1981 & NIAB, Faisalabad \\
\hline 8 & Khushboo-95 & Mutant of Jajai-77 & Aromatic & 1996 & NIA, Tandojam, Sindh \\
\hline 9 & Lateefy & IR760/Basmati-370 & Aromatic & 1983 & RRI, Dokri, Sindh \\
\hline 10 & Rachna-basmati & Basmati-370 SC Variant & Aromatic & 1996 & NARC, Islamabad \\
\hline 11 & Shaheen-basmati & Super-Basmati/Basmati-385 & Aromatic & 2001 & SSRl, Pindi Bhatian \\
\hline 12 & Sonahri-sugdasi & Local selection & Aromatic & 1952 & RRI, Dokri, Sindh \\
\hline 13 & Sugdasi-bengalo & Introduction from Bengal & Aromatic & 1942 & RRI, Dokri, Sindh \\
\hline 14 & Sugdasi-ratria & Local selection & Aromatic & 1956 & RRI, Dokri, Sindh \\
\hline 15 & Sugdasi-sadagulab & Local selection & Aromatic & 1945 & RRI, Dokri, Sindh \\
\hline 16 & Super-basmati & Basmati-370/10486 & Aromatic & 1996 & RRI, KSK, Lahore \\
\hline 17 & Dilrosh-97 & & Non-aromatic & 1997 & ARI, Mingora, Swat \\
\hline 18 & DR-82 & BU1/CR-115 & Non-aromatic & 1982 & RRI, Dokri, Sindh \\
\hline 19 & DR-83 & IR1833/C4-63 & Non-aromatic & 1983 & RRI, Dokri, Sindh \\
\hline 20 & DR-92 & IR8/IET1039-2-4 & Non-aromatic & 1993 & RRI, Dokri, Sindh \\
\hline 21 & Fakhre-Malakand & $\begin{array}{c}\text { Taichung Sen-10//IR29/ I-Geo- } \\
\text { Gen//Taichung Sen-10 }\end{array}$ & Non-aromatic & 2006 & ARI, Mingora, Swat \\
\hline 22 & IR6 (Mehran-69) & Siam29/DGWG & Non-aromatic & 1971 & RRI, Dokri, Sindh \\
\hline 23 & IR36 & IR2042/CR9413 & Non-aromatic & 1976 & IRRI, Philippines \\
\hline 24 & Jhona-349 & Local selection & Non-aromatic & 1933 & RRI, KSK, Lahore \\
\hline 25 & Kangni-27 & Pure line selection & Non-aromatic & 1932 & RRI, Dokri, Sindh \\
\hline 26 & Kanwal-95 & Selection from CN540 & Non-aromatic & 1998 & RRI, Dokri, Sindh \\
\hline 27 & $\mathrm{KS}-282$ & Basmati-370/IR95 & Non-aromatic & 1982 & RRI, KSK, Lahore \\
\hline 28 & NIAB-IR9 & Mutant of IR6 & Non-aromatic & 2001 & NIAB, Faisalabad \\
\hline 29 & Pak23710 & Advanced breeding line & Non-aromatic & - & RRI, KSK, Lahore \\
\hline 30 & Pakhal & $\begin{array}{c}\text { IR36/IR10154-23-3-3 } \\
\text { //R9129-209-2-2-1 }\end{array}$ & Non-aromatic & 1993 & ARS, Mansehra \\
\hline 31 & Palman-suffaid & Local selection & Non-aromatic & 1939 & RRI, KSK, Lahore \\
\hline 32 & Sada-hayat & PTB33/IR4432-53 & Non-aromatic & 1988 & RRI, Dokri, Sindh \\
\hline 33 & Sarshar & Mutant of IR8 & Non-aromatic & 2001 & NIA, Tandojam, Sindh \\
\hline 34 & Sathra & Local selection & Non-aromatic & 1934 & RRI, KSK, Lahore \\
\hline 35 & Shadab & Mutant of IR6 & Non-aromatic & 1999 & NIA, Tandojam, Sindh \\
\hline 36 & Shua-92 & Mutant of IR8 & Non-aromatic & 1993 & NIA, Tandojam, Sindh \\
\hline 37 & Swat-1 & $\mathrm{JP5} / \mathrm{YRL}$ & Non-aromatic & 1984 & ARI, Mingora, Swat \\
\hline 38 & Swat-2 & CR126-42-2/IR2061-2/3 & Non-aromatic & 1984 & ARI, Mingora, Swat \\
\hline 39 & Kinmaze & Ryosaku/AichnakateAsahi & Japonica & 1948 & Japan \\
\hline 40 & Nipponbare & Yamabiko/Sachikaze & Japonica & 1961 & Japan \\
\hline
\end{tabular}

*ARI = Agricultural Research Institute, Mingora, Swat, Pakistan;

ARS: Agricultural Research Station, Dhodial, Mansehra, Pakistan;

NARC: National Agricultural Research Center, Islamabad, Pakistan;

NIA: Nuclear Institute of Agriculture, Tandojam, Sindh, Pakistan;

NIAB: Nuclear Institute for Agriculture \& Biology, Faisalabad, Pakistan;

RRI: Rice Research Institute, Dokri, Sindh, Pakistan;

RRI, KSK: Rice Research Institute, Kala Shah Kaku, Lahore, Pakistan;

SSRI: Soil Salinity Research Institute, Pindi Bhattian, Hafizabad, Pakistan. 
Inc.), 1 unit DNA polymerase (AmpliTaq Gold) and $20 \mathrm{ng}$ of template DNA. Amplifications were performed in a GeneAmp PCR System 9700 (PE Applied Biosystems, USA). Thermal cycler was programmed to 1 cycle of 5 min at $94^{\circ} \mathrm{C}$ for initial strand separation. This was followed by 45 cycles of $1 \mathrm{~min}$ at $94^{\circ} \mathrm{C}$ for denaturation, $1 \mathrm{~min}$ at $36^{\circ} \mathrm{C}$ for annealing and $2 \mathrm{~min}$ at $72^{\circ} \mathrm{C}$ for primer extension. Finally, 1 cycle of $7 \mathrm{~min}$ at $72^{\circ} \mathrm{C}$ was used for final extension, followed by soaking at $4^{\circ} \mathrm{C}$. The reproducibility of the amplification products was checked twice for each primer.

After amplification, the aliquots of $15 \mu \mathrm{l}$ of PCR products plus loading dye were loaded into $1.5 \%$ agarose gels for electrophoresis in 1xTBE (10 mM Tris-Borate, $1 \mathrm{mM}$ EDTA) buffer containing $0.5 \mu \mathrm{g} \mathrm{ml}^{-1}$ of ethidium bromide. The $1 \mathrm{~kb}$ plus was used as a molecular size marker. After electrophoresis, the gels were documented using a FluorChem FC2 Imaging System (Alpha Innotech Corporation, USA).

\section{Data analysis}

Each DNA fragment amplified by a given primer was treated as a unit character and the RAPD fragments were scored as present (1) or absent (0) of the primer-cultivar combinations. Since DNA samples consisted of a bulk sample of DNA extracted from 5 10 seeds, a low intensity for any particular fragment may be explained by the lesser representation of that particular sequence in the bulk sample. Therefore, the intensity of the bands was not taken into account and the fragments with the identical mobility were considered to be the identical fragments. Only major bands consistently amplified were scored and faint bands were not considered. The molecular size of the amplification products was calculated from a standard curve based on the known size of DNA fragments of a $1 \mathrm{~kb}$ plus molecular size weight marker. Pair-wise comparisons of the cultivars based on the presence or absence of unique and shared amplification products were used to generate similarity coefficients. Estimates of genetic similarity (F) were calculated between all pairs of the cultivars according to Nei and Li (1979) based on following formula:

Similarity $(F)=2 N_{a b} /\left(N_{a}+N_{b}\right)$

Where $\mathrm{N}_{\mathrm{a}}=$ the total number of fragments detected in individual ' $a$ '; $\mathrm{N}_{\mathrm{b}}=$ the total number of fragments shown by individual ' $\mathrm{b}$ ' and $\mathrm{N}_{\mathrm{ab}}$ = the number of fragments shared by individuals ' $a$ ' and ' $b$ '.

The resulting similarity coefficients were used to evaluate the relationships among traditional varieties and improved cultivars with a cluster analysis using an unweighted pairgroup method with arithmetic averages (UPGMA). The analysis was plotted in the form of a dendrogram. All computations were carried out using the NTSYS-pc, Version 2.2 package (Rohlf, 2005).

\section{RESULTS}

\section{DNA amplification and cultivar identification}

The genetic diversity and the relationships among rice genotypes were evaluated by RAPD markers using 25 primers. Figure 1 shows the amplification profiles generated with the primer OPA-18 across 40 traditional and improved cultivars. A considerable level of variability was observed among different cultivars. In most cases, aromatic rice exhibited similar banding patterns. The RAPDs exhibited several bands that were shared among the basmati and fine cultivars, whereas a few bands were shared among 'indica' and 'japonica' cultivars of rice. Two japonica cultivars 'Kinmaze' and 'Nipponbare' shared a limited number of bands with all the other cultivars showing more distant relationship to other indica rice varieties. The cultivars 'Jhnoa-349' and 'Lateefy' also displayed unique pattern in comparison to all other genotypes. Interestingly, many primers revealed characteristic fragments in these two cultivars which were not produced in any of the other cultivars, while 'IR6' and 'IR36' had unique as well as shared fragments with the other rice cultivars from Pakistan.

Among the 40 decamer oligonucleotide primers, 25 were chosen. There were 4 primers that did not amplify the DNA in some of the cultivars, 8 primers had resulted in faint bands and 3 primers gave monomorphic banding patterns, hence, they were eliminated from the analysis. Each of the remaining 25 primers varied greatly in their ability to resolve variability among cultivars. Some primers generated several markers, while others generated only a few. A total of 208 reproducible and scorable amplification products were generated across 40 cultivars (Table 2).

The number of amplification products generated by each primer varied from 4 (OPA-15) to 16 (OPB-17) with an average of 8.3 bands primer $^{-1}$. A total of 186 (89.4\%) polymorphic bands were observed ranging from 2 to 15 fragments primer $^{-1}$. The primer OPA-17 and OPB-17 gave the highest number of polymorphic fragments (15), while the minimum number of polymorphic bands (2) using OPA-05 primer. The average number of polymorphic fragments per primer among the 40 rice cultivars was 7.4. The size of the amplified fragments had ranged from 200 (OPA-08) to 4000 bp (OPA-20). The study showed that most of the basmati cultivars genetically resembled each other. 'Jhona-349', 'Lateefy' and 'Swat-1' were distinct from all other aromatic as well as non-aromatic cultivars.

\section{Similarity matrix}

A similarity matrix based on the proportion of shared RAPD fragments was used to establish the level of relatedness between the traditional and improved cultivars. Pair-wise estimates of similarity ranged from 0.50 to 0.96 (Table 3). Two traditional cultivars 'Sugdasi-sadagulab' and 'Sonahri-sugdasi' were the closest genotypes with the 
Table 2. Primers used for generating RAPDs in traditional and improved cultivars of rice from Pakistan.

\begin{tabular}{|c|c|c|c|c|c|c|}
\hline SI. & Primer's & Sequence & Amplified & Polymorphic & Percent & Fragment \\
\hline 1 & OPA-01 & CAGGCCCTTC & 5 & 3 & 60 & $350-2000$ \\
\hline 2 & OPA-02 & TGCCGAGCTG & 5 & 4 & 80 & $400-1350$ \\
\hline 3 & OPA-04 & AATCGGGCTG & 10 & 8 & 80 & $500-1600$ \\
\hline 4 & OPA-05 & AGGGGTCTTG & 5 & 2 & 40 & $500-1500$ \\
\hline 5 & OPA-07 & GAAACGGGTG & 8 & 8 & 100 & $300-1600$ \\
\hline 6 & OPA-08 & GTGACGTAGG & 10 & 10 & 100 & $200-1800$ \\
\hline 7 & OPA-09 & GGGTAACGCC & 8 & 7 & 88 & $450-1450$ \\
\hline 8 & OPA-10 & GTGATCGCAG & 7 & 6 & 86 & $400-1000$ \\
\hline 9 & OPA-11 & CAATCGCCGT & 6 & 6 & 100 & $350-900$ \\
\hline 10 & OPA-12 & TCGGCGATAG & 6 & 6 & 100 & $400-1600$ \\
\hline 11 & OPA-13 & CAGCACCCAC & 8 & 6 & 75 & $425-1400$ \\
\hline 12 & OPA-15 & TTCCGAACCC & 4 & 3 & 75 & $400-1250$ \\
\hline 13 & OPA-16 & AGCCAGCGAA & 5 & 5 & 100 & $500-1200$ \\
\hline 14 & OPA-17 & GACCGCTTGT & 15 & 15 & 100 & $550-3000$ \\
\hline 15 & OPA-18 & AGGTGACCGT & 11 & 10 & 91 & $400-1650$ \\
\hline 16 & OPA-19 & CAAACGTCGG & 12 & 12 & 100 & $300-3000$ \\
\hline 17 & OPA-20 & GTTGCGATCC & 12 & 11 & 92 & $550-4000$ \\
\hline 18 & OPB-06 & TGCTCTGCCC & 6 & 5 & 83 & $650-3000$ \\
\hline 19 & OPB-07 & GGTGACGCAG & 8 & 8 & 100 & $500-3000$ \\
\hline 20 & OPB-08 & GTCCACACGG & 12 & 10 & 83 & $350-2300$ \\
\hline 21 & OPB-11 & GTAGACCCGT & 9 & 9 & 100 & $500-2000$ \\
\hline 22 & OPB-12 & CCTTGACGCA & 5 & 3 & 60 & $700-2000$ \\
\hline 23 & OPB-13 & TTCCCCCGCT & 8 & 7 & 88 & $500-4000$ \\
\hline 24 & OPB-17 & AGGGAACGAG & 16 & 15 & 94 & $350-3500$ \\
\hline 25 & OPB-18 & CCACAGCAGT & 7 & 7 & 100 & $600-3100$ \\
\hline \multicolumn{3}{|c|}{ Total } & 208 & 186 & & \\
\hline
\end{tabular}

highest similarity index of 96\%. This was followed by $91 \%$ similarity between a pair of improved cultivars 'Shadab' and 'Sarshar'. The lowest level of similarity (50\%) was obtained between 'NIAB-IR9' and 'Kinmaze'. The genetic distances between the aromatic cultivars were comparatively low. When the two japonica cultivars; 'Kinmaze' and 'Nipponbare' were excluded from the analysis, the similarity coefficients among the remaining cultivars had ranged between 0.52 and 0.96 . When aromatic cultivars were compared with non-aromatic types, aromatic cultivars were found to be more similar to each other than to non-aromatic group.

Among the aromatic cultivars, similarity coefficients had ranged from 0.62 to 0.96 , whereas non-aromatic cultivars showed similarity coefficients of 0.54 to 0.91 . Although comparison of aromatic cultivars indicated that these were closely related, no cultivar was exactly identical to any other cultivar. Of the cultivars analyzed, 'Kinmaze' and 'Nipponbare' had the greatest dissimilarity with all the 
other tested cultivars. The similarity coefficients of 'Kinmaze' with all the other indica cultivars had ranged from 0.50 to 0.73 , while 'Nipponbare' showed the similarity coefficients of 0.56 to 0.75 with all the other genotypes. Similarly, the similarity coefficients of 'Jhona349 ' and 'Swat- 1 ' with other cultivars had ranged from 0.60 to 0.74 and 0.60 to 0.76 , respectively, while both showed 0.60 similarity index with each other.

\section{Cluster analysis}

Genetic similarities obtained from RAPD data were used to create a cluster diagram. Cluster analysis based on Nei and Li's similarity coefficients using UPGMA grouped 40 cultivars into 3 main clusters I, II and III corresponding to aromatic, non-aromatic and japonica type, respectively and a few independent cultivars (Figure 2). Cluster analysis had placed most of the traditional tall, long-grained aromatic cultivars together showing a high level of genetic relatedness. However, the clusters formed by the aromatic cultivars were distinct from those of non-aromatic and japonica types. Both japonica cultivars did not fall into any of the indica groups and formed a separate cluster which was more distant from all the aromatic and coarse cultivars. Similarly, 'Swat-2' was grouped with japonica cultivars instead of other indica cultivars. Two traditional cultivars 'Jhona-349' and 'Lateefy' also formed two independent clusters which were isolated from all the aromatic as well as non-aromatic cultivars. Interestingly, a number of traditional and improved cultivars originating from various parts of Pakistan did not form a well and defined groups. Moreover, they were interspersed with one another in the cluster analysis indicating no association between the RAPD patterns and the geographic origin of cultivars.

\section{DISCUSSION}

Genetic erosion and habitat destruction by modern agriculture has increased the significance of collection and preservation of plant germplasm. In many rice growing countries, the landraces carrying a vast amount of genetic diversity were distributed in remote villages. The number of landraces began to decline in 1970's when high-yielding varieties were introduced. Most of the old landraces are now available in certain gene banks only, not in the hands of farmers. In Pakistan, collection of native rice varieties was initiated in 1972 over the whole country, and more than 1000 accessions were collected. They are now preserved by the PARC but not available in their original habitats. It is essential to rationalize conservation and use of genetic resources to guide in the establishment of strategies that ensure the maintenance of genetic variability, essential in plant breeding.

In the present investigation, RAPD markers were employed to assess the genetic diversity among 40 traditional and improved cultivars of rice from Pakistan. Although the use of molecular markers to study the genetic diversity and relationships among the different cultivars has been previously reported (Davierwala et al. 2000; Porreca et al. 2001; Neeraja et al. 2002; Saker et al. 2005), the information on the genetic relationship between Pakistani cultivars is limited.The results of present study indicated a considerable level of genetic variation in the cultivars. RAPDs produced a number of fragments that were shared among the basmati and other fine cultivars, whereas comparatively lower number of bands was common among indica and japonica cultivars of rice.

Cultivars 'Kinmaze' and 'Nipponbare' shared limited number of fragments with all the other cultivars, presenting their distant association with indica cultivars. The RAPD assay generated variety-specific products in some of the genotypes. These may be used as DNA fingerprints for variety identification. It would be of immense use for the establishment of proprietary rights and the determination of cultivar purity. There were reports of the numerous unique markers in scented rice germplasm and Egyptian rice cultivars (Raghunathachari et al. 2000; Saker et al. 2005). They further suggested the application of unique markers in DNA typing and variety identification. It would be especially valuable if some of the RAPD markers could be linked to agronomically-important traits.

Twenty-five decamer primers used in RAPD analysis of 40 genotypes could the average number of polymorphic bands per primer was 7.4. This percentage of polymorphic bands was similar to that observed in other rice studies using RAPD markers. Among the seven Egyptian rice genotypes 7.8 polymorphic bands primer ${ }^{-1}$ were detected (Saker et al. 2005). While an average of 6.7 polymorphic fragments perimer $^{-1}$ were observed among AA genome species of rice (Ren et al. 2003).

The proportion of polymorphic fragments was relatively higher as expressed by arbitrary primers as detected 2.7, 4.4, 4.0 and 5.4 polymorphic fragments primer $^{-1}$ (Virk et al. 1995; Parsons et al. 1999; Davierwala et al. 2000; Choudhury et al. 2001).However, the proportion of polymorphic fragments was comparatively lower than that earlier reports as observed on an average of 10.2, 13.7 and 14.0 polymorphic bands primer $^{-1}$ using Indian scented basmati and Italian rice cultivars (Verma et al. 1999; Raghunathachari et al. 2000; Porreca et al. 2001). This discrepancy may relate to genotypes and the selection of RAPD primers with scorable bands. The other reason could be the use of more diverse genotypes.

Similarity coefficients among various cultivars ranged from 0.50 to 0.96 were presented. Similar values of similarity coefficients were obtained among 42 Indian elite rice varieties with average similarity value of 0.83 indicating a narrower genetic variability in the improved varieties (Davierwala et al. 2000). Likewise, similarity coefficients ranging from 0.59 to 0.92 were observed in AA-genome Oryza species from different locations for the RAPD analysis (Ren et al. 2003). However, the value of 0.90 is relatively high when compared to the reports of other 
Table 3. Genetic similarity coefficients among 40 traditional and improved cultivars of rice from Pakistan.

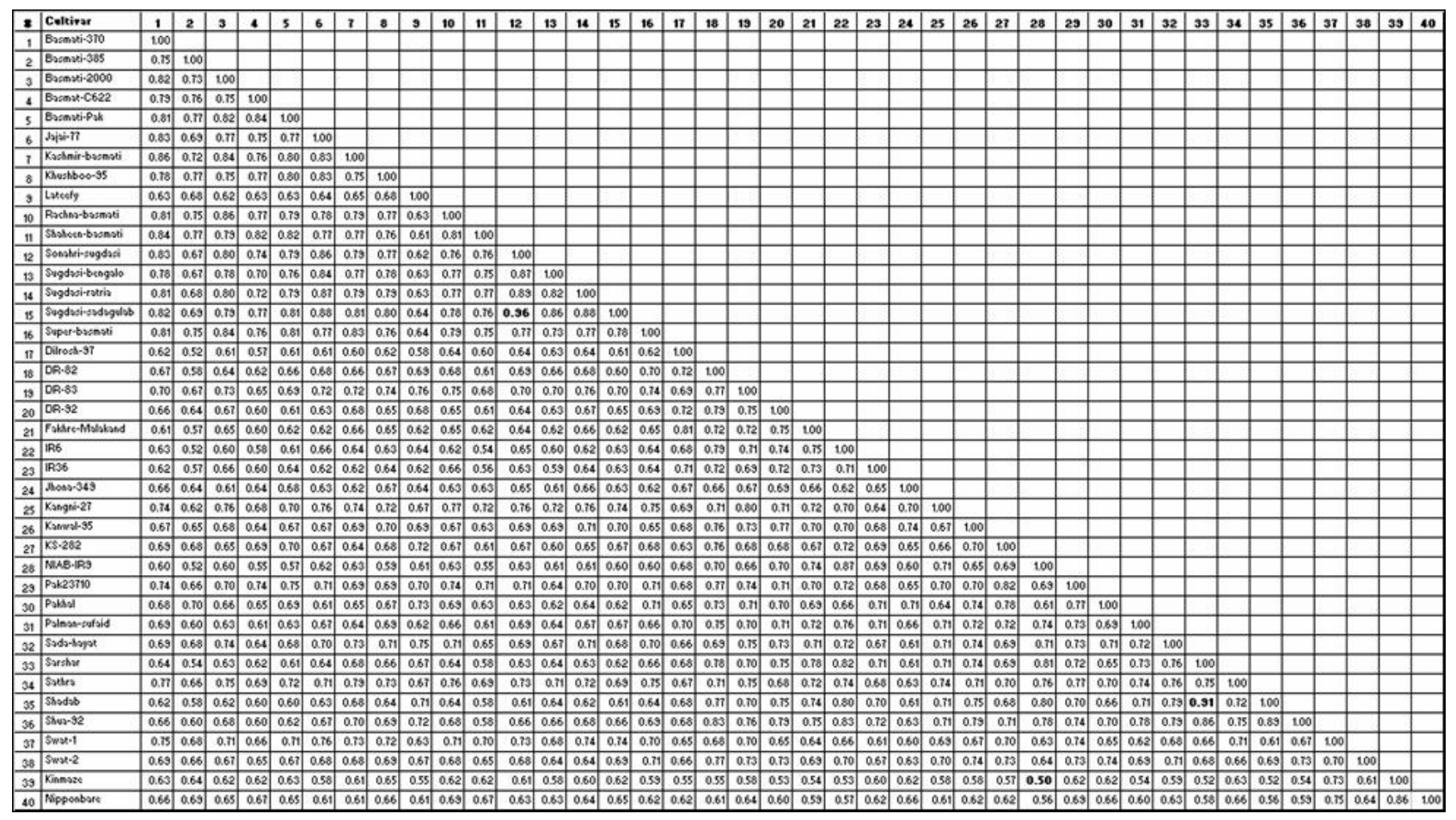

RAPD studies that obtained 25 to $77.5 \%$ genetic similarity among scented rice cultivars from India (Raghunathachari et al. 2000).The high level of similarity could be the result of the intra-specific variations in Pakistani rice due to the common ancestor and the selection of similar traits as compared to Indian scented rice germplasm.

The genetic diversity analysis helped in genotypic grouping of rice types and subspecies level. In the cluster analysis, most of the aromatic cultivars fell into a close sub-group corresponding to pedigree, center of origin and grain type. For example, 15 of the 16 aromatic cultivars, except 'Lateefy' were grouped into the upper portion of the dendrogram of which is consistent with grain quality (aromatic type) as a trait while the rice quality could differentiate cultivars into broad categories.

Cluster analysis grouped 5 aromatic rice from Sindh; 'Jajai77', 'Sugdasi-ratria', 'Sugdasi-sadagulab', 'Sugdasibengalo' and 'Sonahri-sugdasi' into a local selection of Sugdasi rice of which belonging to Rice Research Institute, Dokri, Sindh. They possibly have a common ancestor. Similarly, most of the basmati cultivars including 'Basmati2000', 'Kashmir-basmati', 'Basmati-Pak', 'Basmati-C622' and 'Super-basmati' were clustered indicating that they are more genetically similar compared to other rice cultivars. These rice cultivars shared the genetics of 'Basmati-370' as one of the parent cultivar in their pedigree.

In the similar type of study previously conducted using RAPD markers, the long-grain basmati cultivars were grouped into a significant cluster, whereas the other shortgrained aromatic rice fell into a different group (Choudhury et al. 2001; Arif et al. 2005). They reported a low level of variability among the Indian and Pakistani genotypes of basmati rice using RAPD markers. Similarly, there was a report that classified the indica and japonica cultivars into a separate group and clustering was less pronounced within the japonica group (Mackill, 1995). A close association was also observed among the Indian scented basmati germplam in a previous study using RAPD markers (Davierwala et al. 2000; Choudhury et al. 2001). A low level of genetic variability was detected among the commercial Australian and USA rice lines and their relatives due to a narrow genetic base using RAPD makers (Ko et al. 1994).

In the non-aromatic group, 'Shua-92', 'Shadab' and 'Sarshar' were clustered because they were developed by Nuclear Institute of Agriculture, Tandojam and they are the mutants of either IR6 or IR8. Moreover, they displayed similar plant type and morphological characteristics. IR6 was also associated with this cluster. Two traditional cultivars, 'Jhona-349' and 'Lateefy' appeared to be genetically distinct due to their lower level of similarity with all the cultivars. Likewise, 'Swat-1' was exceptionally diverse and did not fall into any of the 2 main groups of Pakistani rice. It exhibited the lowest similarity with all cultivars and formed a separate group with japonica rice. Two japonica cultivars; 'Kinmaze' and 'Nipponbare' were both showing an $86 \%$ similarity, however they had a low level of similarity with the aromatic cultivars. Interestingly, various cultivars belonging to different parts of Pakistan 
were intermingled with one another in the cluster analysis and did not form well-defined diverse groups. This demonstrated no relationship between the RAPD profiles and the geographic origin of the cultivars.

In the investigation, it is concluded that the aromatic rice cultivars of Pakistan had a relative narrowing genetic base as compared to non-aromatic types. Lower level of polymorphisms in aromatic cultivars indicated that there is a basic similarity among the basmati genotypes which is due to the common ancestor and the similarity of the selective traits. Aromatic cultivars showed a close morphological relation. In the present study, it also indicated that the genetics of the basmati rice is dissimilar from the japonica type. Furthermore, the above results demonstrated that the pattern of variations revealed by RAPD had closely reflected the previously understood relationships between the rice cultivars. Our results can be used as a baseline of relationships for future diversity assessment of rice in Pakistan and the RAPD technique is a fast, efficient method for variability assessment that currently used in genetic resourcesmanagement.

\section{ACKNOWLEDGMENTS}

We are indebted to various Rice Research Institutes/Programs in Pakistan for providing the seed material of their traditional and improved cultivars of rice. We would also like to acknowledge the seed of japonica cultivars of rice provided by the National Institute of Agrobiological Sciences, Tsukuba, Japan. We gratefully acknowledge the financial support for this work from the Agricultural Linkages Program under the AREP.

\section{REFERENCES}

ARIF, M.; KOUSAR, S.; BAJWA, M.A.; ARIF, A. and ZAFAR, Y. Genetic diversity among rice genotypes of Pakistan through random amplified polymorphic DNA (RAPD) analysis. Pakistan Journal of Botany, September 2005, vol. 37, no. 3, p. 585-592.

BAUTISTA, N.S.; SOLIS, R.; KAMIJIMA, O. and ISHII, T. RAPD, RFLP and SSLP analyses of phylogenetic relationships between cultivated and wild species of rice. Genes and Genetic Systems, May 2001, vol. 76, no. 2, p. 71-79.

CHOUDHURY, P.R.; KOHLI, S.; SRINIVASAN, K.; MOHAPATRA, T. and SHARMA, R.P. Identification and classification of aromatic rices based on DNA fingerprinting. Euphytica, April 2001, vol. 118, no. 3, p. 243-251.

DAVIERWALA, A.P.; CHOWDARI, K.V.; KUMAR, S.; REDDY, A.P.K.; RANJEKAR, P.K. and GUPTA, V.S. Use of three different marker systems to estimate genetic diversity of Indian elite rice varieties. Genetica, May 2000, vol. 108, no. 3, p. 269-284.
KANG, H.W.; CHO, Y.G.; YOON, U.H. and EUN, M.Y. A rapid DNA extraction method for RFLP and PCR analysis from a single dry seed. Plant Molecular Biology Reporter, March 1998, vol. 16, no. 1, p. 1-9.

KO, H.I.; COWAN, D.C.; HENRY, R.J.; GRAHAM, G.C.; BLAKENEY, A.B. and LEWIN, L.G. Random amplified polymorphic DNA analysis of Australian rice (Oryza sativa L.) varieties. Euphytica, January 1994, vol. 80, no. 3, p. 179-189.

MACKILL, D.J. Classifying japonica rice cultivars with RAPD markers. Crop Science, 1995, vol. 35, no. 3, p. 889894.

NEERAJA, C.N.; SARLA, N. and SIDDIQ, E.A. RAPD analysis of genetic diversity in Indian landraces of rice (Oryza sativa L). Journal of Plant Biochemistry and Biotechnology, 2002, vol. 11, p. 93-97.

NEI, M. and LI, W. Mathematical model for studying genetic variation in terms of restriction endonucleases. Proceedings of the National Academy of Sciences of the United States of America, October 1979, vol. 76, no. 10, p. 5269-5273.

O'NEILL, R.; SNOWDON, R.J. and KOHLER, W. Population genetics aspects of biodiversity. Progress in Botany, 2003, vol. 64, p. 115-137.

PARSONS, B.J.; NEWBURY, H.J.; JACKSON, M.T. and FORD-LLOYD, B.V. The genetic structure and conservation of aus, aman and boro rices from Bangladesh. Genetic Resources and Crop Evolution, December 1999, vol. 46, no. 6, p. 587-598.

PORRECA, P.; SABINA, M.R.; MARTELLI, G.; SUNSERI, F.; GRECO, I.; PRUNEDDU, G. and SPANU, A. Genetic variability among Italian rice (Oryza sativa L.) cultivars investigated by RAPDs analysis. Journal of Genetics \& Breeding, 2001, vol. 55, p. 349-355.

RAGHUNATHACHARI, P.; KHANNA, V.K.; SINGH, U.S. and SINGH, N.K. RAPD analysis of genetic variability in Indian scented rice germplasm (Oryza sativa L.). Current Science, October 2000, vol. 79, no. 7, p. 994998.

REN, F.; LU, B.; LI, S.; HUANG, J. and ZHU, Y. A comparative study of genetic relationships among the AAgenome Oryza species using RAPD and SSR markers. Theoretical and Applied Genetics, December 2003, vol. 108, no. 1, p. 113-120.

ROHLF, F. J. NTSYSpc (Numerical Taxonomy and Multivariate Analysis System). Version 2.2, 2005, Exeter Software, Applied Biostatistics Inc., New York, USA.

SAKER, M.M.; YOUSSEF, S.S.; ABDALLAH, N.A.; BASHANDY, H.S. and EL-SHARKAWY, A.M. Genetic 
analysis of some Egyptian rice genotypes using RAPD, SSR and AFLP. African Journal of Biotechnology, September 2005, vol. 4, no. 9, p. 882-890.

SASAKI, T. and BURR, B. International rice genome sequence project: the effort to complete the sequence of rice genome. Current Opinion in Plant Biology, April 2000, vol. 3, no. 2, p. 138-141.

VERMA, S.K.; KHANNA, V. and SINGH, N. Random amplified polymorphic DNA analysis of Indian scented basmati rice (Oryza sativa L.) germplasm for identification of variability and duplicate accessions, if any. Electrophoresis, June 1999, vol. 20, no. 8, p. 1786-1789.

VIRK, P.S.; NEWBURY, H.J.; JACKSON, M.T. and FORD-LLOYD, B.V. The identification of duplicate accessions within a rice germplasm collection using RAPD analysis. Theoretical and Applied Genetics, June 1995, vol. 90, no. 7-8, p. 1049-1055.

WILLIAMS, J.G.K.; KUBELIK, A.R.; LIVAK, K.J.; RAFALSKI, J.A. and TINGEY, S.V. DNA polymorphisms amplified by arbitrary primers are useful as genetic markers. Nucleic Acids Research, 1990, vol. 18, no. 22, p. 6531-6535. 